\title{
Article
}

\section{Causes of Variation in Food Preference in the Netherlands}

\author{
Jacqueline M. Vink ${ }^{1, \star}$, Kirsten J. M. van Hooijdonk ${ }^{1, \star}$, Gonneke Willemsen ${ }^{2}$, Edith J. M. Feskens ${ }^{3}$ and Dorret I. Boomsma ${ }^{2}$ \\ ${ }^{1}$ Behavioural Science Institute, Radboud University, Nijmegen, The Netherlands, ${ }^{2}$ Department of Biological Psychology, Vrije Universiteit Amsterdam, Amsterdam, \\ The Netherlands and ${ }^{3}$ Division of Nutrition and Health, Wageningen University, Wageningen, The Netherlands
}

\begin{abstract}
Our current society is characterized by an increased availability of industrially processed foods with high salt, fat and sugar content. How is it that some people prefer these unhealthy foods while others prefer more healthy foods? It is suggested that both genetic and environmental factors play a role. The aim of this study was to (1) identify food preference clusters in the largest twin-family study into food preference to date and (2) determine the relative contribution of genetic and environmental factors to individual differences in food preference in the Netherlands. Principal component analysis was performed to identify the preference clusters by using data on food liking/disliking from 16,541 adult multiples and their family members. To estimate the heritability of food preference, the data of 7833 twins were used in structural equation models. We identified seven food preference clusters (Meat, Fish, Fruits, Vegetables, Savory snacks, Sweet snacks and Spices) and one cluster with Drinks. Broad-sense heritability (additive [A] + dominant [D] genetic factors) for these clusters varied between .36 and .60. Dominant genetic effects were found for the clusters Fruit, Fish (males only) and Spices. Quantitative sex differences were found for Meat, Fish and Savory snacks and Drinks. To conclude, our study convincingly showed that genetic factors play a significant role in food preference. A next important step is to identify these genes because genetic vulnerability for food preference is expected to be linked to actual food consumption and different diet-related disorders.
\end{abstract}

Keywords: Food preference; liking; heritability; twin-family

(Received 9 June 2020; accepted 9 July 2020)

Some people prefer sweet food, while others prefer savory food. Some people will choose healthy snacks, while others will choose unhealthy snacks. How is it that individuals differ in their food preferences and food choice? Although food preferences are thought to form early in life (Nicklaus \& Schwartz, 2019) and persist into adulthood (Nicklaus et al., 2005), they can develop and change during the lifespan (Anzman-Frasca et al., 2018). Food preferences are shaped by many factors, including physiological, nutritional, environmental and sociocultural factors. Our current society is characterized by an increased availability of industrially processed foods with high salt, fat and sugar content. The easy availability of this type of food has caused a shift from eating for survival (energy intake) to more hedonic eating (feelings of reward; Peters et al., 2002). Generally, the most palatable, and therefore pleasurable, foods are both energy dense and high in fat content (Drewnowski, 1998; Stubbs \& Whybrow, 2004). As expected, individual food preference is correlated with reported food intake (correlations between .58 and .86; Drewnowski et al., 1999).

Food preferences are often assessed by using food hedonic questionnaires. These questionnaires determine the degree of 'liking' or 'wanting' a specific product (Zoghbi, 2016; Zoghbi et al., 2019).

Author for correspondence: Jacqueline M. Vink, Email: J.vink@bsi.ru.nl

*Shared first authors.

Cite this article: Vink JM, van Hooijdonk KJM, Willemsen G, Feskens EJM, and Boomsma DI. (2020) Causes of Variation in Food Preference in the Netherlands. Twin Research and Human Genetics 23: 195-203, https://doi.org/10.1017/thg.2020.66
The literature reports food preference clusters, including fruits and/or vegetables, meat and fish (high-protein foods) and snacks (sweet and savory snacks) (Breen et al., 2006; Drewnowski et al., 1999; Fildes et al., 2014; Johnson et al., 2014; Pallister et al., 2015; Smith et al., 2016; Tornwall et al., 2014; Wardle et al., 2001). Some studies have also reported clusters of dairy foods (Fildes et al., 2014; Smith et al., 2016), starch foods (Fildes et al., 2014; Smith et al., 2016) or distinctive tastes (Pallister et al., 2015).

Food preference and taste intensity have been shown to differ across gender and age. Males have been shown to prefer warm, hearty, meal-related comfort foods (such as steak, casseroles and soup), while females instead preferred comfort foods that were more snack-related (such as chocolate and ice cream; Wansink et al., 2003). Younger people preferred more snack-related comfort foods (such as potato chips, ice-cream or cookies), while those over 55 years of age preferred more meal-related foods (such as soup, burgers or side-dishes) (Wansink et al., 2003). A study on perceived taste intensity of salt, sour, bitter and sweet showed that males (compared to females) perceived weaker taste intensity. Additionally, older (compared to younger) participants perceived stronger taste intensity (Fischer et al., 2013).

The contribution of genetic and environmental factors to individual differences in food preferences of different food clusters has been investigated in $4 \mathrm{UK}$ studies, representing 3 independent samples with sample sizes varying between 331 and 2865 participants. The heritability of liking vegetables and fruits was found to be moderate, with estimates varying between .36 and .54 . For meat

(c) The Author(s), 2020. Published by Cambridge University Press. This is an Open Access article, distributed under the terms of the Creative Commons Attribution-NonCommercialNoDerivatives licence (http://creativecommons.org/licenses/by-nc-nd/4.0/), which permits non-commercial re-use, distribution, and reproduction in any medium, provided the original work is unaltered and is properly cited. The written permission of Cambridge University Press must be obtained for commercial re-use or in order to create a derivative work. 


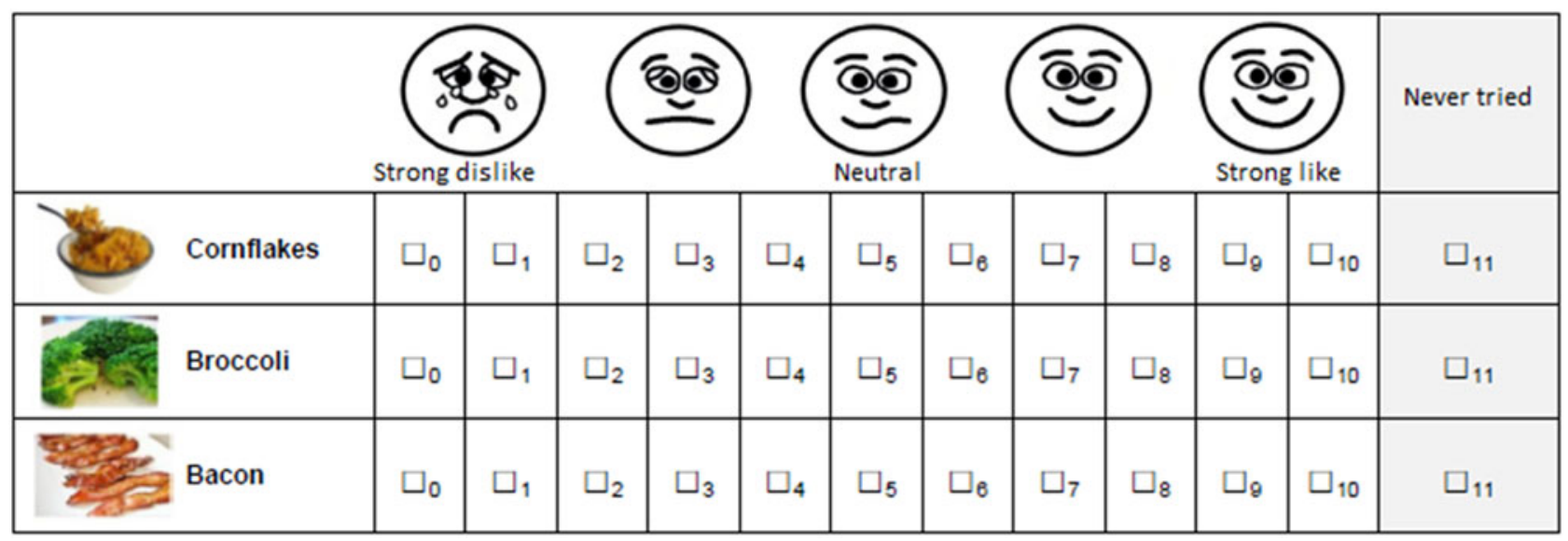

Fig. 1. The example of items used in the food preference questionnaire. '0' indicates strong dislike, ' 10 ' indicates strong like and ' 11 ' indicates that the participant has not tried this item. Questionnaire is adapted from Duffy et al. (2007).

and fish, heritability estimates were somewhat higher, varying between .44 and .78, while the heritability of snacks was suggested to be low-to-moderate, varying between .20 and .52 . The heritability estimates in the two studies including children (Breen et al., 2006; Fildes et al., 2014) were comparable to heritability estimates in the two studies including adults (Pallister et al., 2015; Smith et al., 2016). The studies regarding children found a significant influence of common environmental effects on the phenotypic variance in food preference, while in adults no contribution of the common environment was identified. So far as only UK samples were studied, it is important to also study samples from other countries, as food preference clusters and the relative contribution of genetic factors (heritability) might vary as a result of cultural or environmental differences.

The aim of the current study is to (1) identify food preference clusters in the largest adult twin study into food preference to date $(N=16,541$ individuals from twin-family sample) and (2) determine the relative contribution of genetic and environmental factors to individual differences in food preference in the Netherlands ( $N=7833$ twins).

\section{Materials and Methods}

\section{Setting and Subjects}

The sample with food preference data $(N=16,541)$ consisted of adult twins and multiples $(n=8280)$ and their parents $(n=5685)$, siblings $(n=1643)$, spouses $(n=668)$, offspring of multiples $(n=249)$ and other family members $(n=16)$ registered at the Netherlands Twin Register (NTR; age range: 11-93 years). The NTR was founded in 1987 and collects data about Dutch twins and their families on health, lifestyle and personality (Boomsma et al., 2006; Willemsen et al., 2013). Starting in January 2015, the NTR invited 64,383 members via email or post to complete a questionnaire focussing on food preference. To further increase the response rate, reminders were sent until October 2015.

To estimate the heritability of food preference, the data of a subsample were analyzed, which included twins and in the case of higher order multiples, the first and second born with information on zygosity $(n=7833)$. Fifty-three twins were excluded from analyses due to missing information on zygosity. The zygosity of the same-sex twins was determined by DNA markers or the response on the standard survey questions about physical similarity between the twins (Ligthart et al., 2019). In total, 1087 monozygotic males (MZM; 338 complete pairs), 633 dizygotic males (DZM; 165 complete pairs), 2929 monozygotic females (MZF; 1079 complete pairs), 1407 dizygotic females (DZF; 425 complete pairs), 914 male-female opposite sex twins (DOSmf; 213 complete pairs) and 863 female-male opposite sex twins (DOSfm; 221 complete pairs) were included in the genetic analyses.

\section{Assessment of Food Preference}

The food preference questionnaire was a modified version of the food preference questionnaire developed by Duffy et al. (2007). This questionnaire uses a hedonic liking-disliking scale to assess food preference and determines the degree of 'liking' or 'wanting' a specific product (Zoghbi, 2016; Zoghbi et al., 2019). The questionnaire includes 103 items, of which 71 are food items, 14 are beverage items and 18 are activity items. Some adaptations were made to suit the Dutch population: (1) the questionnaire was translated into Dutch; (2) a few items were changed to suit the Dutch Population (e.g., cheddar cheese was changed to old cheese); (3) the liking-disliking scale was changed from a -100 to +100 rating scale to a $0-10$ category liking-disliking scale ranging from strong dislike (0) to strong like (10); (4) an additional category was added where participants could indicate that they had never tried a specific food item and (5) a digital version of the questionnaire was developed. Figure 1 provides an example of items used in the food preference questionnaire. In addition to this preference questionnaire, participants also provided information on characteristics such as age, height, weight, general health status, smoking behavior and educational level.

\section{Statistical Methods}

Demographic characteristics. Statistical analyses were performed in IBM SPSS Statistics 25 and in the Mx Software (Neale et al., 1999). Descriptive statistics were obtained for sex and age of participants, general health state (ranging between 0 , poor health state and 5, perfect health state), body mass index (BMI; weight $/$ height $^{2}$ ), educational level (low: lower general secondary education, lower vocational education; middle: higher general secondary education, intermediate vocational education and high: higher vocational education, university and post-graduate 
16,541 participants completed the questionnaire

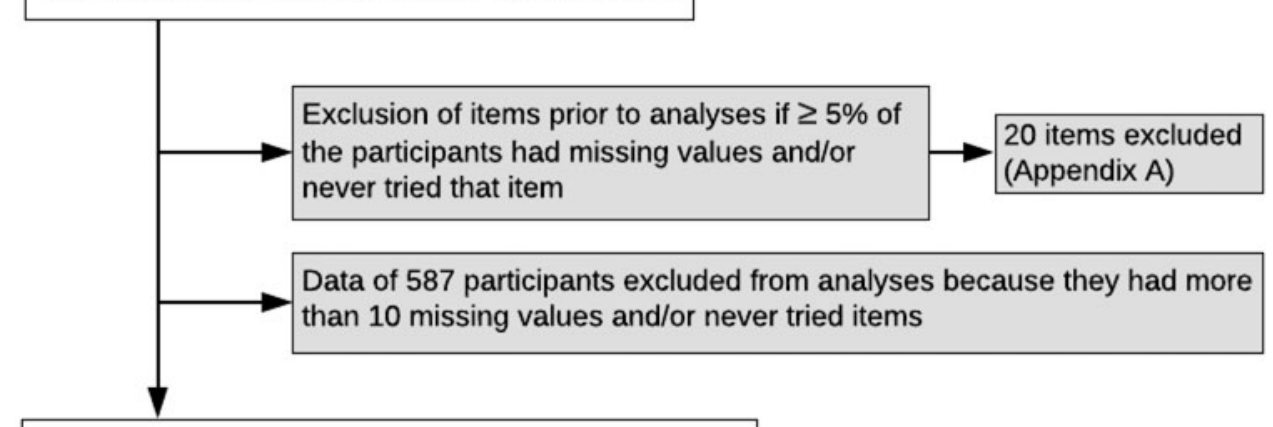

15,954 participants eligible for further analyses

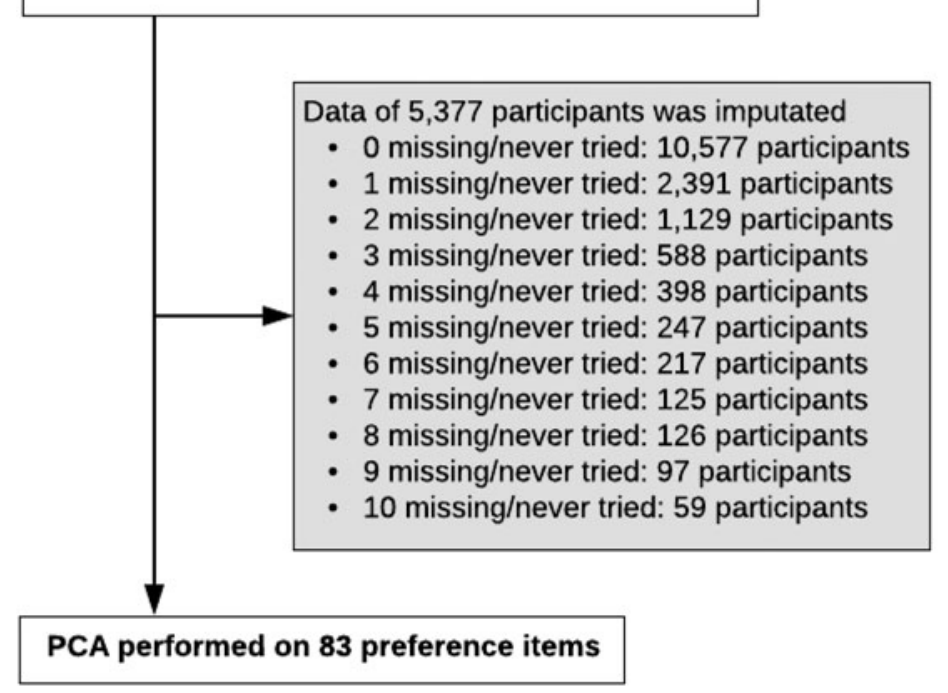

Fig. 2. Study pipeline PCA.

education or $\mathrm{PhD}$ ) and smoking status (if the participant ever or never smoked on a regular base).

Food preference cluster analysis. Principal component analysis (PCA) was performed to identify the preference clusters by using data from multiples and their family members. Figure 2 summarizes the study pipeline. First, 20 of 103 items were excluded because more than $5 \%$ of the participants had missing data (not answered) and/or had not tried that item (see Supplementary file A). Secondly, data from 587 participants were excluded from further analyses because these participants had 11 or more included items 'never tried' or missing. Therefore, data from 15,954 participants were eligible for further analyses. Imputation was required for participants with 10 or less missing and/or never tried items. For 5377 participants, the mean of each item for either males or females was imputated. PCA was performed including 83 preference items by use of the varimax rotation method because data were orthogonal. The number of components was determined by examining the inflection point in the scree plot.

Model fitting analyses - twin correlations. Structural equation modeling in $\mathrm{Mx}$ software was used for genetic analyses (Neale et al., 1999). First, twin correlations (indicating the resemblance between twin pairs) for five different zygosity groups (MZM, DZM, MZF, DZF and DOS) were estimated (= saturated model).

Next, we tested whether there was: (1) an effect of zygosity, (2) an effect of sex or (3) an effect of age on the mean. We also tested whether there was: (4) an effect of zygosity or (5) an effect of sex on the variance. Finally, we tested whether (6) covariances were equal across sexes. This last step was only executed when an effect of zygosity and an effect of sex on the variance were identified. The submodels were tested by using the means of likelihood-ratio tests. The negative log-likelihood $(-2 \mathrm{LL})$ was subtracted from the general model and compared with the restricted model. The output provides a chi-squared $\left(\chi^{2}\right)$ with degrees of freedom $(d f)$ of the difference in $-2 \mathrm{LL}$ scores of the general model and the restricted model. When the $\chi^{2}$ test resulted in a $p$-value higher than .01 , the restricted model was comparable to the general model and was chosen as the most parsimonious model. This model is used for comparison to the next restricted model. If the $\chi^{2}$ test resulted in a $p$-value lower than .01 , the restricted model was not deemed to be the most parsimonious model.

Model fitting analyses - ADE modeling (heritability estimates). Heritability was estimated for each preference cluster by genetic structural equation modeling (Neale et al., 1999). These models decomposed variation due to additive genetic effects (A), common environmental effects (C), dominance genetic effects (D) and nonshared environmental effects (E). In studies including only MZ and DZ twins, the contributions of common environmental effects and dominance genetic effects cannot be estimated simultaneously, and a choice needs to be made for one of these components based on the twin correlation pattern. There is an evidence of additive genetic effects when the correlation of MZ twins 
Table 1. Identified food preference clusters after PCA

\begin{tabular}{lcl}
\hline Clusters & $\begin{array}{c}\text { Explained } \\
\text { variance (\%) }\end{array}$ & Included food preference items \\
\hline Meat & 12.17 & Pork chops, ham, chargrilled meats, sausage, crispy bacon, baked chicken, beef steak and fried chicken (eight items). \\
\hline Fish & 1.67 & Tuna or salmon, prawns, shellfish and fried fish (three items). \\
\hline Fruits & 8.76 & Pear, strawberries, yellow or green melon, pineapple, cherries, banana and orange juice (seven items). \\
\hline Vegetables & 1.95 & Broccoli, spinach or greens, beetroot and lentils or beans (four items). \\
\hline Savory snacks & 4.84 & Chips, tortilla chips or crisp, pizza and mayonnaise (four items). \\
\hline Sweet snacks & 3.68 & Biscuits, cakes or pastries, cheesecake, cake icing, ice cream, jam or jelly and high-fiber bar (six items). \\
\hline Spices & 2.39 & Chili pepper, burn of spicy, black pepper, garlic and raw onion (five items). \\
\hline Drinks & 2.13 & Red wine, beer, white wine, black coffee and going to the pub/bar (five items). \\
\hline Sport & 2.96 & Playing sports, exercising with others, working up a sweat, exercising alone, taking the stairs and bicycling (six items). \\
\hline
\end{tabular}

is higher than the correlation of DZ twins. Common environmental effects are suggested when the correlation of DZ twins is more than half the correlation of MZ twins. Dominance genetic effects are suggested when the correlation of the DZ twins is less than half the correlation of $\mathrm{MZ}$ twins. Unique environmental influences reflect the environmental factors not shared by twin pairs (and also include error). The relative contribution of additive genetic effects on the variance in a trait is referred to as Narrow-Sense heritability. Broad-Sense heritability reflects the variation in a trait due to all sources of genetic variance, that is, additive genetic and dominance genetic effects (Knopik et al., 2016). In this study, an ADE model was fitted to the data based on the correlation pattern given in the Results section.

Next, we investigated whether sex differences in covariance structure were present. When testing for qualitative sex differences, we tested whether there were different genetic factors in males and females that influence food preference. When testing quantitative sex differences, we tested whether the additive genetic effects, dominance genetic effects and nonshared environmental effects were of equal size for males and females. Additionally, the dominance genetic effects (model 3) and additive genetic effects and dominance genetic effects (model 4) were constrained to zero to test if, respectively, AE or E models were more parsimonious than the ADE models. Models were again compared with each other using a $\chi^{2}$ test. The same strategy as mentioned above in 'Model fitting analyses - twin correlations' was used to determine the most parsimonious model.

Spouse correlations. Spouses are genetically unrelated but share their environment and consequently eat together. To explore the similarity in spouse pairs, we calculated the spouse correlations (father and mother of twins as well as twin and their spouse) for all food clusters with Pearson correlations. If both members of a twin pairs and their spouses participated, we randomly selected one twin-spouse pair ( $n=78$ pairs excluded).

\section{Ethical Approval}

The Medical Research Ethics Committee of the VU University Medical Centre approved the data collection protocol. An NTR collaboration agreement was signed by the Department of Developmental Psychopathology of the Behavioral Science Institute of the Radboud University Nijmegen with respect to data access and sharing.

\section{Results}

\section{Demographic Characteristics}

About two-thirds of the total sample were female (64.8\%) and more than half $(58.1 \%)$ was highly educated. The mean age of the sample was 44 years, and the sample was healthy (self-reported health score of 4.1 on a scale of 1-5). The mean BMI was 24.5 (SD 4.2 ) and $37.5 \%$ of the sample had overweight (BMI $\geq 25)$. About $40 \%$ of the sample had ever smoked. The total sample $(N=15,954)$ and subsample of multiples $(n=7833)$ were largely comparable in study characteristics. The mean age in the subsample of multiples (35.1 years) was slightly lower than the mean age in the total sample (44.1 years). Even though the mean BMI was comparable between both samples, fewer people were overweight $(\mathrm{BMI} \geq 25)$ in the subsample of multiples (37.5\% vs. $25.8 \%)$.

\section{Food Preference Cluster Analysis}

We identified seven food preference clusters (Meat, Fish, Fruits, Vegetables, Savory snacks, Sweet snacks and Spices, explained variance $35 \%$ ), one cluster with Drinks (explained variance $2 \%$ ) and one cluster with preference for sporty behavior (explained variance $3 \%$; Table 2). This last cluster will not be included in the remaining analyses because it is not related to preferences for food or drinks. Supplementary Table S1 provides all included items in the PCA with a mean liking-disliking score, standard deviation and factor loadings. Supplementary Figure S1 B includes the scree plot.

\section{Association Between Sample Characteristics and Preference Clusters}

For most clusters, the liking scores of men and women were comparable (Table 1). The largest difference was seen for liking of Meat, where men scored on average 10 points higher than women (53.6 for men, 43.9 for women). Men scored also higher on liking of Spices (27.4 vs. 24.1 ), while women scored higher on the liking of Fruit (50.5 for men vs. 53.0 for women). Age was positively associated with liking of Meat, Fish, Vegetables and negatively with Savory snacks, Sweet snacks and Drinks. BMI was positively associated with a preference for Meat and negatively associated with a preference for Fruits, Savory snacks, Sweet snacks and Drinks. Though correlations between BMI and the food preference clusters were significant, they were in general low $(<.10)$. 


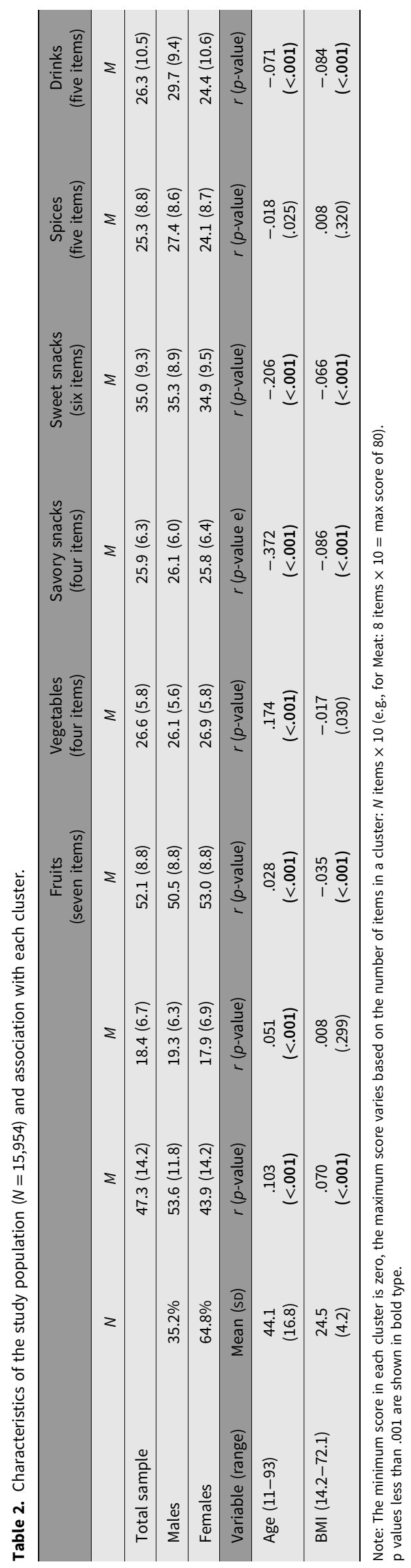

\section{Model Fitting Analyses - Twin Correlations}

In Table 3, the twin correlations from the saturated model and the most parsimonious model per preference cluster are provided. The results of all full and restricted models for the food and drink preference clusters are given in Supplementary Table S2. Both sex (model 2) and age (model 3) had a significant effect on the mean for all preference clusters, with a few exceptions. There was no sex effect for the cluster Sweet snacks and no age effect for the cluster Fruits and Spices.

In all clusters, the $\mathrm{MZ}$ correlations were higher than the $\mathrm{DZ}$ correlations. In most clusters, the $\mathrm{DZ}$ correlation was less than half of the MZ correlation, and therefore ADE models were fitted to the data.

\section{Model Fitting Analyses - ADE Modeling (Heritability Estimates)}

In all eight clusters, the contribution of genetic factors was significant. Broad-sense heritability varied between .36 and .60 (Table 4, more model fitting details in Supplementary Table S3). We did not find qualitative sex differences for any of the clusters, but we did find quantitative sex differences for the food clusters Meat, Fish and Savory snacks and the nonfood cluster Drinks. Dominant genetic effects were significantly present for the clusters Fruits, Fish (males only) and Spices. For the other clusters an $\mathrm{AE}$ model fitted the data best.

\section{Spouse Correlations}

The correlations between spouse pairs (both spouse pairs consisting of parents of twins as well as spouse pairs consisting of a twin with his/her spouse) was significant for all clusters except Fruits (not significant in twin-spouse pairs), varying between .12 and .31 (see Supplementary Table S4).

\section{Discussions}

This is the first Dutch study, and the largest study worldwide to identify food preference patterns and determine to which extent these patterns are influenced by genetic and environmental factors. We identified seven food preference clusters, including Meat, Fish, Fruits, Vegetables, Savory snacks, Sweet snacks and Spices, and one Drinks cluster. The broad-sense heritability of these clusters varied between 36\% for the cluster Savory snacks and $60 \%$ for the clusters Fish and Drinks.

Six of the seven identified food clusters were also reported in previous studies on food preference. Only the cluster Spices has not been reported before. Other studies also reported clusters of Dairy products (Fildes et al., 2014; Smith et al., 2016), Starch foods (Fildes et al., 2014; Smith et al., 2016), and Distinctive tastes (Pallister et al., 2015), but these were not identified in the current study. Inconsistency with the previous literature might be explained by the lack of power in some previous studies (due to smaller sample size), and methodological differences or cultural differences between participants in previous studies and Dutch participants in the current study. It must be noted that several items that fall under 'Distinctive tastes' (e.g., wasabi, fresh coriander, tabasco sauce, blue cheese, eggplant, curries) were excluded from the analyses in our study because the percentage of people who did not answer the question or who indicated to have never tried it was more than 5\%. Therefore, it was not possible to find a cluster with Distinctive tastes. This could reflect a cultural difference between the UK and Dutch population. 
Table 3. Twin correlations from the saturated model and the most parsimonious model per food preference cluster

\begin{tabular}{|c|c|c|c|}
\hline & & Saturated model & Most parsimonious model \\
\hline Cluster & Zygosity & $\begin{array}{c}\text { Correlation } \\
{[95 \% \mathrm{CI}]}\end{array}$ & $\begin{array}{c}\text { Correlation } \\
{[95 \% \mathrm{CI}]}\end{array}$ \\
\hline \multirow[t]{5}{*}{ Meat } & MZM & $.46[.37, .54]$ & $.46[.37, .54]$ \\
\hline & DZM & $.19[.04, .33]$ & $.19[.04, .33]$ \\
\hline & MZF & $.53[.50, .57]$ & $.53[.49, .57]$ \\
\hline & DZF & $.16[.07, .25]$ & $.16[.07, .25]$ \\
\hline & DOS & $.11[.02, .20]$ & $.11[.02, .20]$ \\
\hline \multirow[t]{5}{*}{ Fish } & MZM & $.61[.54, .68]$ & $.60[.54, .66]$ \\
\hline & DZM & $.23[.08, .37]$ & $.25[.09, .39]$ \\
\hline & MZF & $.53[.48, .57]$ & $.54[.50, .58]$ \\
\hline & DZF & $.15[.06, .24]$ & $.14[.05, .22]$ \\
\hline & DOS & $.25[.16, .33]$ & $.25[.16, .33]$ \\
\hline \multirow[t]{5}{*}{ Fruits } & MZM & $.35[.25, .44]$ & $.43[.39, .47]$ \\
\hline & DZM & $.30[.15, .43]$ & $.11[.05, .17]$ \\
\hline & MZF & $.45[.40, .49]$ & $.43[.39, .47]$ \\
\hline & DZF & $.11[.02, .21]$ & $.11[.05, .17]$ \\
\hline & DOS & $.03[-.06, .13]$ & $.11[.05, .17]$ \\
\hline \multirow[t]{5}{*}{ Vegetables } & MZM & $.54[.46, .61]$ & $.52[.48, .55]$ \\
\hline & DZM & $.24[.09, .38]$ & $.24[.19, .30]$ \\
\hline & MZF & $.51[.47, .56]$ & $.52[.48, .55]$ \\
\hline & DZF & $.25[.16, .34]$ & $.24[.19, .30]$ \\
\hline & DOS & $.23[.14, .32]$ & $.24[.19, .30]$ \\
\hline \multirow{5}{*}{$\begin{array}{l}\text { Savory } \\
\text { snacks }\end{array}$} & MZM & $.39[.30, .48]$ & $.39[.30, .48]$ \\
\hline & DZM & $.20[.05, .35]$ & $.20[.05, .34]$ \\
\hline & MZF & $.46[.41, .50]$ & $.46[.41, .50]$ \\
\hline & DZF & $.18[.09, .27]$ & $.18[.09, .28]$ \\
\hline & DOS & $.14[.05, .23]$ & $.14[.05, .23]$ \\
\hline \multirow[t]{5}{*}{ Sweet snacks } & MZM & $.45[.36, .53]$ & $.47[.43, .50]$ \\
\hline & DZM & $.10[-.06, .25]$ & $.17[.11, .23]$ \\
\hline & MZF & $.48[.43, .52]$ & $.47[.43, .50]$ \\
\hline & DZF & $.23[.14, .32]$ & $.17[.11, .23]$ \\
\hline & DOS & $.14[.05, .23]$ & $.17[.11, .23]$ \\
\hline \multirow[t]{5}{*}{ Spices } & MZM & $.52[.43, .59]$ & $.53[.49, .56]$ \\
\hline & DZM & $.29[.15, .43]$ & $.17[.11, .23]$ \\
\hline & MZF & $.54[.50, .58]$ & $.53[.49, .56]$ \\
\hline & DZF & $.16[.06, .25]$ & $.17[.11, .23]$ \\
\hline & DOS & $.13[.04, .22]$ & $.17[.11, .23]$ \\
\hline \multirow[t]{5}{*}{ Drinks } & MZM & $.56[.49, .63]$ & $.53[.46, .59]$ \\
\hline & DZM & $.31[.16, .44]$ & $.33[.17, .45]$ \\
\hline & MZF & $.61[.57, .64]$ & $.61[.57, .64]$ \\
\hline & DZF & $.22[.13, .31]$ & $.22[.13, .30]$ \\
\hline & DOS & $.20[.11, .29]$ & $.21[.12, .30]$ \\
\hline
\end{tabular}

Note: MZM, monozygotic male; MZF, monozygotic female; DZM, dizygotic male; DZF, dizygotic female; DOS, dizygotic opposite sex twin; $95 \% \mathrm{Cl}$, 95\% confidence interval.
In agreement with Wansink et al. (2003), we found differences in liking of clusters between men and women. The largest difference observed in the current study was seen for liking of meat. Wansink et al. (2003) also found differences in liking of meat between men and women, while a study of Breen et al. (2006) did not. We also identified a difference between liking of spices (difference of 3.3). This is in line with research suggesting that men more strongly prefer spicy foods compared to women (Logue \& Smith, 1986; Tornwall et al., 2014). Additionally, the negative association between age and liking savory and sweet snacks was also found by Wansink et al. (2003). Whether food preference is associated with BMI is inconclusive. In our study we found significant but very small correlations, while Pallister et al. (2015) found stronger correlation and Johnson et al. (2014) did not observe a significant relationship.

The broad-sense heritability estimates in our study are comparable with results from previous studies (see Supplementary Table S5). These four UK studies (Breen et al., 2006; Fildes et al., 2014; Pallister et al., 2015; Smith et al., 2016) reported heritability estimates varying between .44 and.78 for Meat and Fish, between .36 and .54 for Fruits and Vegetables and between .20 and.52 for Snacks, while we found between .41 and $.60, .43$ and .51 and .36 and.46, respectively. Previous studies into the heritability of food preference did not identify the cluster Spices. We expect that preference for spicy foods is closely related to pleasantness of oral pungency of spicy foods. In a previous study on oral pungency of spicy foods, the heritability estimate was 50\% (Tornwall et al., 2012), which is in line with the broad-sense heritability estimate of $53 \%$ we have identified in the present study for liking-disliking Spices.

Our food preference questionnaire also included items on liking-disliking of drinks. The cluster Drinks includes beer, red and white wine, black coffee and going to the pub. We are not aware of other studies exploring the heritability of liking-disliking of drinks. Food preference is correlated with food intake (Drewnowski et al., 1999; Duffy et al., 2009), so we expect that preference for Drinks is correlated with actual drinking behavior. Literature describes heritability estimates in adults of $39 \%$ for coffee consumption (Vink et al., 2009), 62\% for coffee preference (defined as total cups of coffee per day divided by total cups of coffee and tea per day; Vink et al., 2009) and 53\% for alcohol intake (van Beek et al., 2014). These findings are in line with our heritability estimate for liking-disliking of drinks ( $H^{2}=53 \%$ males, $60 \%$ females).

Interestingly, the pattern of twin correlations did not indicate evidence for shared environmental factors, and therefore we fitted ADE models. Three previous studies on the heritability of food preference in adults modeled either ACE models (Pallister et al., 2015), ADE models (Tornwall et al., 2014) or both (Smith et al., 2016). None of the studies in adults (Pallister et al., 2015; Smith et al., 2016) found indications for common environmental effects on food preference, while one study (Smith et al., 2016) reported dominant genetic effects on Fruit preference. These results are in line with our results: dominant genetic effects (.26-.47) for some clusters (Fruits, Fish in females and Spices in males), but in most clusters the dominant genetic effect was not significant. Two studies exploring the heritability of food preference in children (Fildes et al., 2014) did find effects of common environmental factors suggesting that these common environmental factors do play a role in childhood. In our study, the influence of unique environmental factors was substantial, varying from .40 to .64. A likely factor influencing food preference in adulthood is food preference of the spouse. In childhood, family meals are mostly eaten 
Table 4. ADE models per food preference cluster

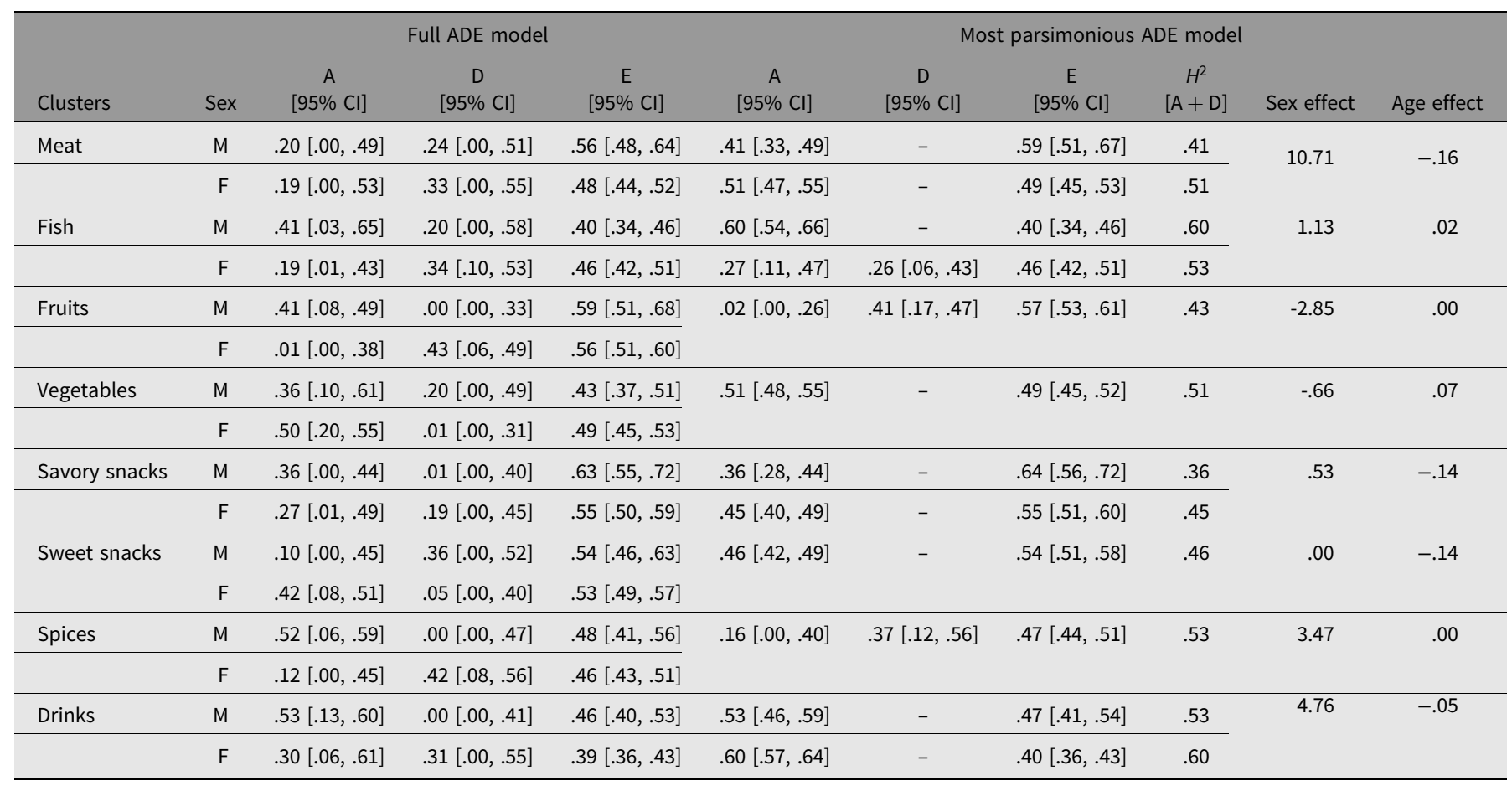

Note: M, males; F, females; A, additive genetic effects; D, dominance genetic effects; E, nonshared environmental effects; $H^{2}$, broad-sense heritability; sex effect, sex effect on the mean food preference score with females $=0$ and males $=1$; age effect, age effect on the mean food preference score.

together with parents and siblings (shared family environment), while in adulthood meals are more often eaten with a spouse (unique environmental influences). This idea is supported by the significant spouse correlations (varying between .12 and .31) for all preference clusters (with one exception of a nonsignificant result for Fruits in twin-spouse pairs). Although speculative, the pattern seems to imply that in children common environment overrules the influence of dominance genetic effects and that these dominance genetic effects come to light in adults (only in some preference clusters like Fruit) when the shared environmental influences decrease. The evidence of dominance genetic effects in adults implies that there is an interaction between the effects of alleles or loci who together influence food preference. Future studies with large sample sizes should aim to confirm the (additive and nonadditive) genetic influences on the food preference in adults.

We did not find a qualitative sex difference in food preference, but we did find quantitative sex differences for the food clusters Meat, Fish and Savory snacks and the nonfood clusters Drinks. Previous, smaller twin studies did not test for sex differences in heritability estimates. For all of the clusters where quantitative sex differences were detected, except Fish, the heritability estimates were slightly higher for females compared with males. This could indicate that for females their genetic make-up plays a slightly larger role in their food preference, while for males the environmental influences are slightly more important. It should be noted that differences were small, and that there were no sex differences for the other four clusters.

\section{Study Strengths and Limitations}

A major strength of this study was the ability to investigate food preference patterns in a non-UK sample, which enabled us to compare heritability estimates between countries. The UK and the Netherlands are both West European countries that might have similar habits regarding food consumption and are comparable in food availability. The generalizability of the results to a non-West European country might be limited and results need to be replicated in studies with a non-West European population. Another strength was the sample size of the current study $(N=15,954$ [twin-family sample], $n=7833$ [subsample of twins]), which is considerably bigger than sample sizes used in previous studies on food preference. Consequently, confidence intervals were narrow, quantitative and qualitative sex differences could be tested, and the influence of nonadditive genetic effects on food preference could be investigated.

A limitation of this study was that only 35 of 71 food preference items were included in a food preference cluster. Some items do not meet the criteria to be included in a food preference cluster, but still did match with the content of a certain cluster. For example, grapefruit had a factor loading of .308 for the cluster Fruit (see Supplementary Table S1), but was not taken into account in this cluster because the correlation was not strong enough (factor loading <.4).

Other limitations apply to the twin models. In these models, we assumed that the equal environment assumption was valid. Violation of these assumptions would result in inflated heritability estimates (Derks et al., 2006; Peyrot et al., 2016). Research has shown that bias due to violation of the equal environment assumption is negligible for traits related to food preference, such as diet, eating disorders, weight and BMI (Felson, 2014; Maes, Neale, Kendler, Hewitt et al., 1998, Maes, Neale, Martin et al., 2006). Therefore, we expect that possible bias is also negligible for food preference. Additionally, random mating is assumed. We did observe significant spouse correlations for food preference 
clusters, which could point to different (not mutually exclusive) mechanisms (Treur et al., 2015). In the case of phenotypic assortment, individuals tend to choose a spouse who is phenotypically similar. With heritable traits like food preference, this could lead to a higher genotypic similarity between spouses (so no random mating). Alternatively, spouses may meet and pair up because they are from similar (social) surroundings (social homogamy) causing spouses to be more similar in food preference. Finally, spousal resemblance may be due to marital interaction, reflecting that two individuals start to resemble each other (with regard to food preference) because they influence each other while being in a relationship together. Under social homogamy and marital interaction, the genetic resemblance between spouses is not expected to be higher (i.e., conform to random mating). So far, it is not known which mechanism explains the observed spouse correlations in our study.

\section{Future Directions}

Our study convincingly showed that genetic factors play a significant role in food preference. A next step should be to quantify the genetic factors for food preference. Based on previous studies, there already are some interesting candidate genes; for example, with regard to taste perception. Humans are able to taste five different flavors as follows: bitter, sweet, sour, salty and umami. For each of the flavors, sets of genes seem to be involved (Chamoun et al., 2018). Regarding bitter taste receptor genes, specifically, taste receptor type 2 gene (T2Rs gene) TAS2R38 has been associated with preference for a variety of products, such as vegetables, berries, coffee, grapefruit, chili, fats and alcohol consumption. Additionally, TAS2R38 variants have been associated with sweet taste preference. Besides TAS2R38 variants, receptors belonging to the T1R or TAS1R family are believed to modulate sweet and umami tastes by interaction with amino acids. The perception of salty tastes is believed to be dependent on the involvement of epithelial sodium channels (ENaCs), which are located in taste cell membranes. The genes SCNN1A, SCNN1B, SCHNN1G and $S C N N 1 D$ code for ENaCs subunits, and variants are believed to influence salty taste perception. The mechanisms behind sour taste sensitivity are not fully understood. However, research suggests that sour taste perception is influenced by acidic foods and substances. Transient receptor potential channels (TRPs), namely polycystic kidney disease like (PKDL-like) receptors, are believed to mediate sour taste. The above-mentioned flavors can partially be linked to the clusters we found: salty tastes links to the cluster Savory snacks, sweet tastes links to the cluster Sweet snacks, and bitter tastes links to the clusters Drinks and Vegetables. Therefore, it is expected that the above-mentioned genes involved in taste perception also play a role in food preference. However, many (more) genes will likely play a role in complex behavior like food preference, so future research should use more advanced methods (i.e., genomewide association studies instead of candidate-gene studies) to identify the genes involve in individual differences in food preference.

It is important to identify these genes because a high- or lowsensitivity of tastes is expected to be linked to actual food consumption and different diet-related disorders. For example, Precone et al. (2019) showed that high-sensitivity of bitter is associated with metabolic diseases, coronary heart disease and aging process and lowsensitivity of bitter is associated with alcohol dependence and aging process. High-sensitivity of sweets is associated with obesity and dental caries, and preference for salty is associated with hypertension and cardiovascular disease. Preference for fatty foods is associated with type 2 diabetes and obesity (Precone et al., 2019). This implies that individuals with a certain combination of genes in favor of fruits and vegetables could more easily follow a healthy diet and resist the temptation to buy unhealthy snacks than individuals with a less favorable set of genes. Our genetically influenced food preferences will likely contribute to differences in food consumption and risk profiles to develop diet-related disorders. This asks for a personalized approach in the field of nutrition epidemiology when genes involved in our food preference are mapped out more thoroughly. This also helps to develop individualized diets that are more likely to be followed.

\section{Conclusions}

This study provides insight into why individuals differ in their food preferences and why it might be easier for some individuals to make healthy food choices compared with other individuals. Future research needs to confirm the results found in this study and explore generalizability to other counties and cultures. Improving our understanding of the origins behind food preference and eating behavior may help to reduce and prevent dietrelated disorders such as obesity, cardiovascular disease, type 2 diabetes and metabolic syndrome via a personalized approach.

Supplementary material. To view supplementary material for this article, please visit https://doi.org/10.1017/thg.2020.66.

Acknowledgments. We thank all members of the the Netherlands Twin Register (NTR) who participated in the food preference survey for their contribution. Data collection for this study was funded by a grant from BBMRI-NL (Biobanking and Biomolecular Resources Research Infrastructure; grant no. 184.021.007 and 184.033.111).

Conflict of interest. None declared.

\section{References}

Anzman-Frasca, S., Ventura, A. K., Ehrenberg, S., \& Myers, K. P. (2018). Promoting healthy food preferences from the start: a narrative review of food preference learning from the prenatal period through early childhood. Obesity Reviews, 19, 576-604.

Boomsma, D. I., de Geus, E. J. C., Vink, J. M., Stubbe, J. H., Distel, M. A., Hottenga, J.-J., ... Willemsen, G. (2006). Netherlands Twin Register: from twins to twin families. Twin Research and Human Genetics, 9, 849-857.

Breen, F. M., Plomin, R., \& Wardle, J. (2006). Heritability of food preferences in young children. Physiology \& Behavior, 88, 443-447

Chamoun, E., Mutch, D. M., Allen-Vercoe, E., Buchholz, A. C., Duncan, A. M., Spriet, L. L., Haines, J., \& Ma, D. W. L. (2018). A review of the associations between single nucleotide polymorphisms in taste receptors, eating behaviors, and health. Critical Reviews in Food Science and Nutrition, 58, 194-207.

Derks, E. M., Dolan, C. V, \& Boomsma, D. I. (2006). A test of the equal environment assumption (EEA) in multivariate twin studies. Twin Research and Human Genetics, 9, 403-411.

Drewnowski, A. (1998). Energy density, palatability, and satiety: implications for weight control. Nutrition Reviews, 56, 347-353.

Drewnowski, A., Henderson, S. A., Levine, A., \& Hann, C. (1999). Taste and food preferences as predictors of dietary practices in young women. Public Health Nutrition, 2, 513-519.

Duffy, V. B., Hayes, J. E., Sullivan, B. S., \& Faghri, P. (2009). Surveying food and beverage liking. Annals of the New York Academy of Sciences, $1170,558-568$.

Duffy, V. B., Lanier, S. A., Hutchins, H. L., Pescatello, L. S., Johnson, M. K., \& Bartoshuk, L. M. (2007). Food preference questionnaire as a screening tool for assessing dietary risk of cardiovascular disease within health risk appraisals. Journal of the American Dietetic Association, 107, 237-245. 
Felson, J. (2014). What can we learn from twin studies? A comprehensive evaluation of the equal environments assumption. Social Science Research, 43, 184-199.

Fildes, A., van Jaarsveld, C. H. M., Llewellyn, C. H., Fisher, A., Cooke, L., \& Wardle, J. (2014). Nature and nurture in children's food preferences. American Journal of Clinical Nutrition, 99, 911-917.

Fischer, M. E., Cruickshanks, K. J., Schubert, C. R., Pinto, A., Klein, B. E. K., Klein, R., ... Snyder, D. J. (2013). Taste intensity in the Beaver Dam Offspring Study. The Laryngoscope, 123, 1399-1404.

Johnson, S. L., Boles, R. E., \& Burger, K. S. (2014). Using participant hedonic ratings of food images to construct data driven food groupings. Appetite, 79, 189-196

Knopik, V. S., Neiderhiser, J. M., DeFries, J. C., \& Plomin, R. (2016). Behavioral genetics (7th ed.). Macmillan Higher Education.

Ligthart, L., van Beijsterveldt, C. E. M., Kevenaar, S. T., de Zeeuw, E., van Bergen, E., Bruins, S., .. Boomsma, D. I. (2019). The Netherlands Twin Register: longitudinal research based on twin and twin-family designs. Twin Research and Human Genetics, 22, 623-636.

Logue, A. W., \& Smith, M. E. (1986). Predictors of food preferences in adult humans. Appetite, 7, 109-125.

Maes, H. H., Neale, M. C., Kendler, K. S., Hewitt, J. K., Silberg, J. L., Foley, D. L., .. Eaves, L. J. (1998). Assortative mating for major psychiatric diagnoses in two population-based samples. Psychological Medicine, 28, 1389-1401.

Maes, H. H., Neale, M. C., Kendler, K. S., Martin, N. G., Heath, A. C., \& Eaves, L. J. (2006). Genetic and cultural transmission of smoking initiation: an extended twin kinship model. Behavior Genetics, 36, 795-808.

Neale, M. C., Boker, S. M., Xie, G., \& Maes, H. H. (1999). MX: Statistical modeling. Richmond, VA: Department of Psychiatry, Virginia Commonwealth University.

Nicklaus, S., Boggio, V., Chabanet, C., \& Issanchou, S. (2005). A prospective study of food variety seeking in childhood, adolescence and early adult life. Appetite, 44, 289-297.

Nicklaus, S., \& Schwartz, C. (2019). Early influencing factors on the development of sensory and food preferences. Current Opinion in Clinical Nutrition and Metabolic Care, 22, 230-235.

Pallister, T., Sharafi, M., Lachance, G., Pirastu, N., Mohney, R. P., MacGregor, A., ... Menni, C. (2015). Food preference patterns in a UK twin cohort. Twin Research and Human Genetics, 18, 793-805

Peters, J. C., Wyatt, H. R., Donahoo, W. T., \& Hill, J. O. (2002). From instinct to intellect: the challenge of maintaining healthy weight in the modern world. Obesity Reviews, 3, 69-74.

Peyrot, W. J., Robinson, M. R., Penninx, B. W. J. H., \& Wray, N. R. (2016). Exploring boundaries for the genetic consequences of assortative mating for psychiatric traits. JAMA Psychiatry, 73, 1189-1195.
Precone, V., Beccari, T., Stuppia, L., Baglivo, M., Paolacci, S., Manara, E., ... Bertelli, M. (2019). Taste, olfactory and texture related genes and food choices: implications on health status. European Review for Medical and Pharmacological Sciences, 23, 1305-1321.

Smith, A. D., Fildes, A., Cooke, L., Herle, M., Shakeshaft, N., Plomin, R., \& Llewellyn, C. (2016). Genetic and environmental influences on food preferences in adolescence. American Journal of Clinical Nutrition, 104, 446-453.

Stubbs, R. J., \& Whybrow, S. (2004). Energy density, diet composition and palatability: influences on overall food energy intake in humans. Physiology \& Behavior, 81, 755-764.

Tornwall, O., Silventoinen, K., Kaprio, J., \& Tuorila, H. (2012). Why do some like it hot? Genetic and environmental contributions to the pleasantness of oral pungency. Physiology \& Behavior, 107, 381-389.

Tornwall, O., Silventoinen, K., Hiekkalinna, T., Perola, M., Tuorila, H., \& Kaprio, J. (2014). Identifying flavor preference subgroups. Genetic basis and related eating behavior traits. Appetite, 75, 1-10.

Treur, J. L., Vink, J. M., Boomsma, D. I., \& Middeldorp, C. M. (2015). Spousal resemblance for smoking: underlying mechanisms and effects of cohort and age. Drug and Alcohol Dependence, 153, 221-228.

van Beek, J. H. D. A., de Moor, M. H. M., Geels, L. M., Willemsen, G., \& Boomsma, D. I. (2014). Explaining individual differences in alcohol intake in adults: Evidence for genetic and cultural transmission? Journal of Studies on Alcohol and Drugs, 75, 201-210.

Vink, J. M., Staphorsius, A. S., \& Boomsma, D. I. (2009). A genetic analysis of coffee consumption in a sample of Dutch twins. Twin Research and Human Genetics, 12, 127-131.

Wansink, B., Cheney, M. M., \& Chan, N. (2003). Exploring comfort food preferences across age and gender. Physiology \& Behavior, 79, 739-747.

Wardle, J., Sanderson, S., Leigh Gibson, E., \& Rapoport, L. (2001). Factoranalytic structure of food preferences in four-year-old children in the UK. Appetite, 37, 217-223.

Willemsen, G., Vink, J. M., Abdellaoui, A., den Braber, A., van Beek, J. H. D. A., Draisma, H. H. M., ... Boomsma, D. I. (2013). The Adult Netherlands Twin Register: twenty-five years of survey and biological data collection. Twin Research and Human Genetics, 16, 271-281.

Zoghbi, M. (2016). Evaluating dietary quality and taste preferences with a simple liking survey: Application to studying individuals with morbid obesity. Honors Scholar Theses, 510. https://opencommons.uconn.edu/ srhonors_theses/510

Zoghbi, M., Stone, A., Papsavas, P., Swede, H., Hubert, P., Tisher, D., \& Duffy, V. B. (2019). Evaluating Taste Preferences and Dietary Quality with a Simple Liking Survey: application in Bariatric Treatment Settings. Bariatric Surgical Practice and Patient Care, 14, 41-48. 